
\title{
28 Research Square \\ Using Nonlinear Dynamics of EEG Signals to Decode Hand Movement Directions Under Bimanual Movement
}

Jiarong Wang

Beijing Institute of Technology

Luzheng Bi ( $\sim$ bhxblz@bit.edu.cn )

School of Mechanical Engineering, Beijing Institute of Technology, Beijing 100081 China https://orcid.org/0000-0001-8986-3379

Weijie Fei

Beijing Institute of Technology

\section{Research}

Keywords: Brain-computer interface, EEG, ESN, Hand movement decoding, Human augmentation, Movement-related cortical potential, Opposite hand motor activity

Posted Date: January 4th, 2021

DOI: https://doi.org/10.21203/rs.3.rs-136558/v1

License: (c) (i) This work is licensed under a Creative Commons Attribution 4.0 International License. Read Full License 


\section{Abstract}

Background: Decoding hand movement parameters from electroencephalograms (EEG) signals can provide intuitive control for brain-computer interfaces (BCls). However, most existing studies of EEGbased hand movement decoding are focused on single hand movement. Since the both-hand movement is common in human augmentation systems, to address the decoding of hand movement under the opposite hand movement, we investigate the neural signatures and decoding of the primary hand movement direction from EEG signals under the opposite hand movement. Methods: The decoding model was developed by using an echo state network (ESN) to extract nonlinear dynamics parameters of movement-related cortical potentials (MRCPs) as decoding features and linear discriminant analysis as a classifier.

Results: Significant differences in MRCPs between movement conditions with and without an opposite hand movement were found. Furthermore, using the ESN-based models, the decoding accuracies reached $86.03 \pm 7.32 \%$ and $88.45 \pm 6.16 \%$ under the conditions without and with the opposite hand movement, 20 respectively.

Conclusions: These findings showed that the proposed method performed well in decoding the primary hand movement directions under the conditions with and without the opposite hand movement. This study may open a new avenue to decode hand movement parameters from EEG signals and lay a foundation for the future development of BCl-based human augmentation systems.

\section{Full Text}

This preprint is available for download as a PDF.

\section{Figures}


(a)

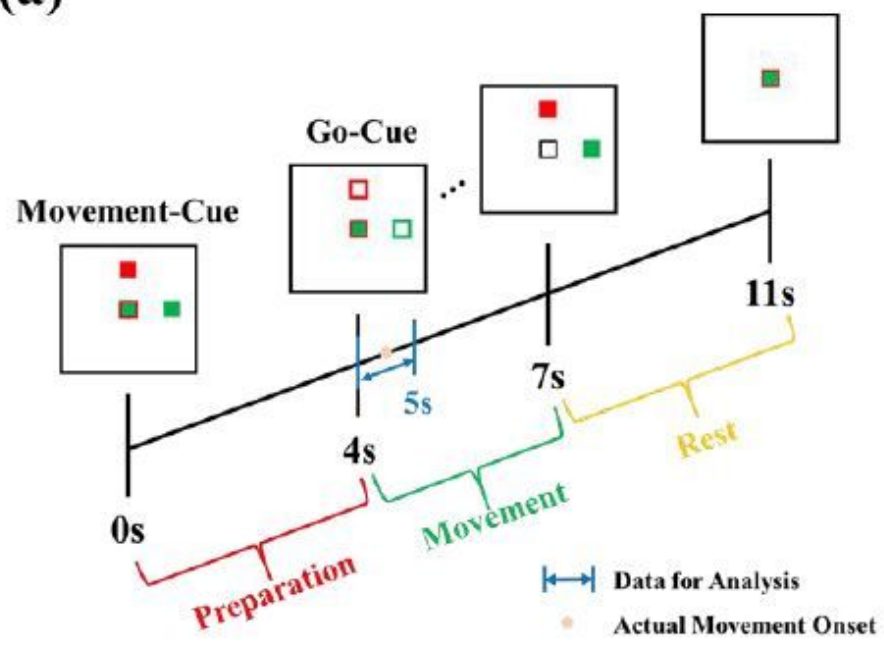

(b)

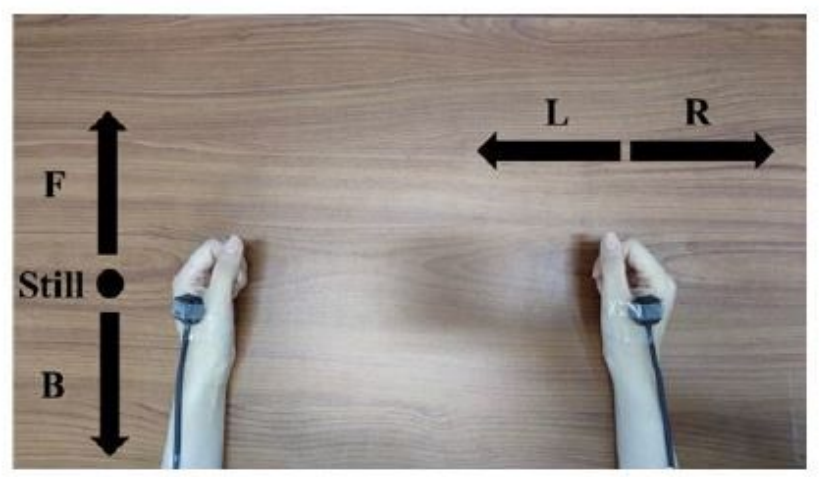

\section{Figure 1}

Experimental Protocol. (a) Timeline of experiment setup. Note that time $0 \mathrm{~s}$ refers to the Movement-Cue, time $4 \mathrm{~s}$ refers to the Go-Cue, and time $4.5 \mathrm{~s}$ is the onset of the actual movement. (b) Illustration of both hand movement directions. Note that ' $F$ ' and ' $B$ ' refer to the movement of left-hand in the forward or backward direction, respectively, and ' $L$ ' and ' $R$ ' refer to the movement of right-hand in the left or right direction, respectively. 


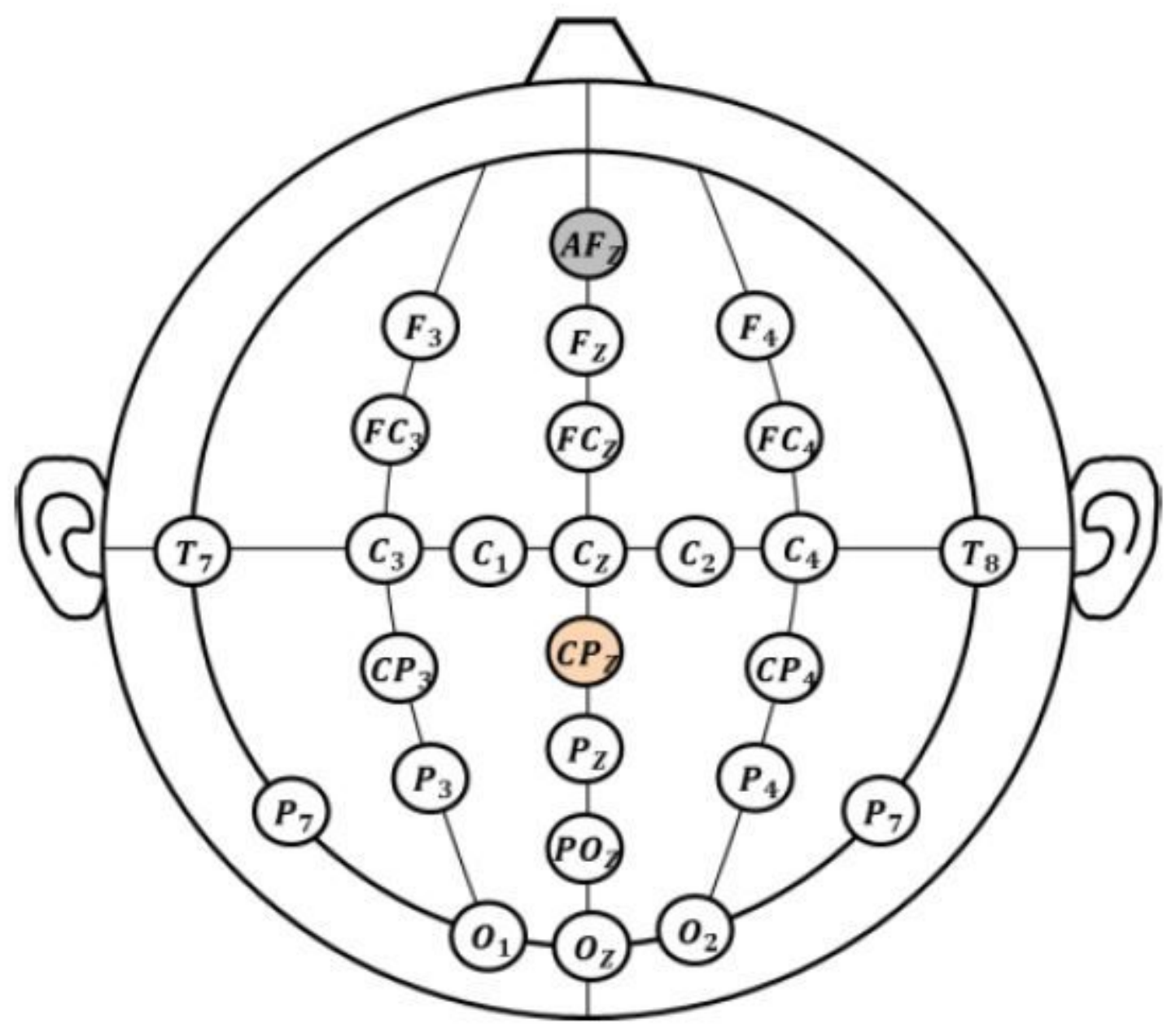

Figure 2

Used Electrodes Position Diagram.

(a)

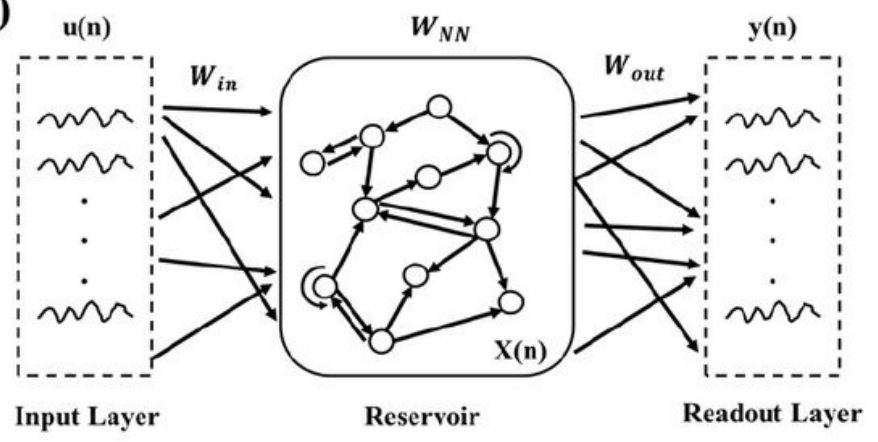

(b)

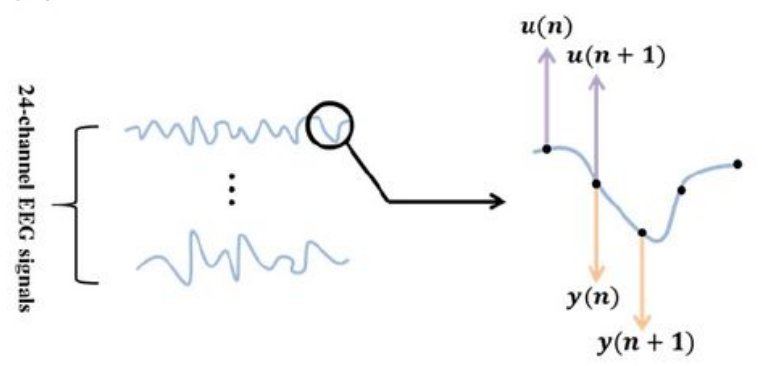

Figure 3

ESN schematic diagram. (a) Network structure diagram. (b) Illustration of the input and output data selection based on the time series of EEG signals. $u(\bullet)$ refers to the input data and $y(\bullet)$ refers to the 
output data.

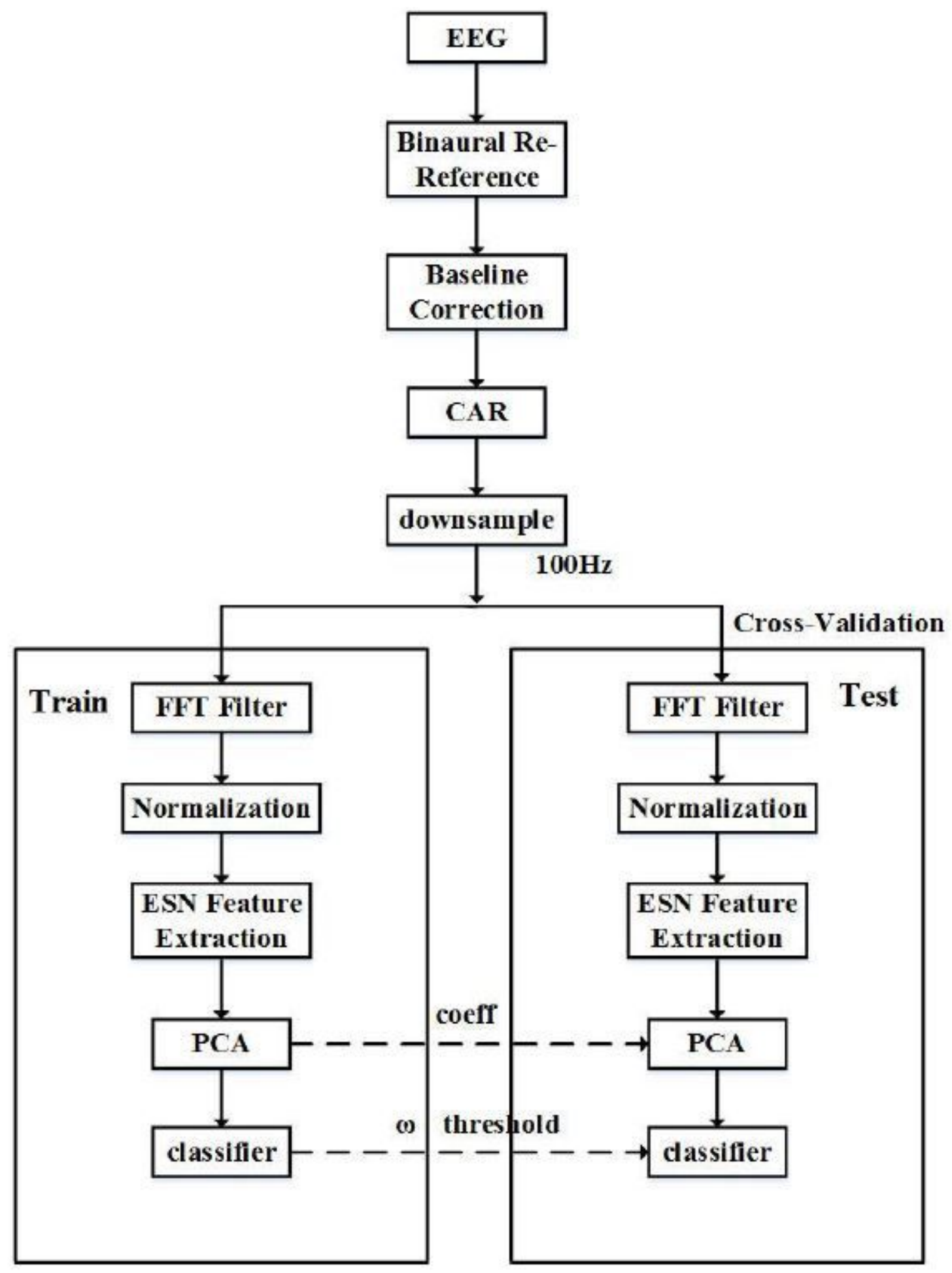

Figure 4

Flow chart of the proposed decoding method. 


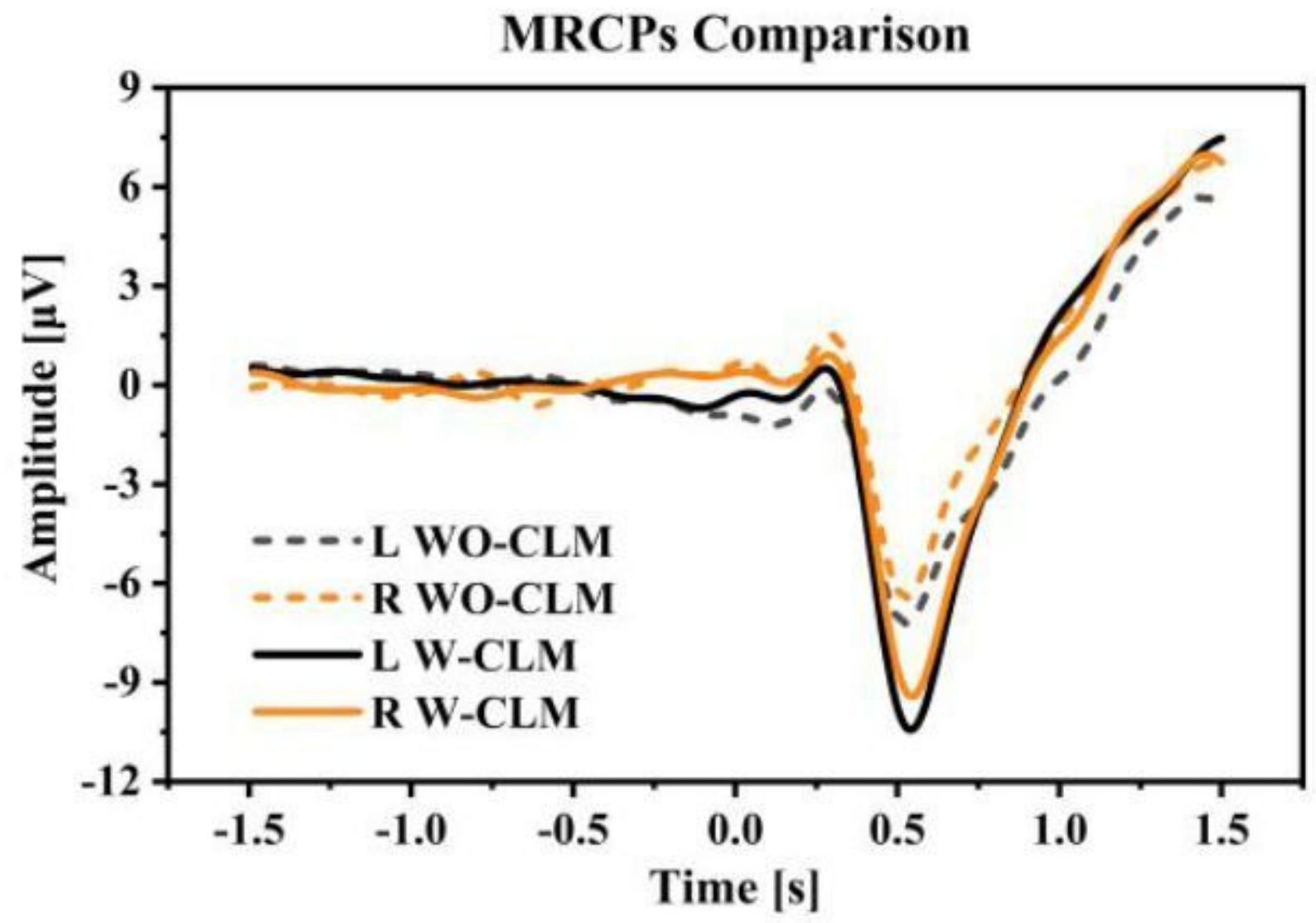

Figure 5

The averaged MRCP at $\mathrm{Cz}$ channel from $-1.5 \mathrm{~s}$ to $1.5 \mathrm{~s}$ of Go-Cue. "L" 371 and "R" refer to the right-hand movement in right and left directions. Note that time $0 \mathrm{~s}$ refers to the Go-Cue, and time $0.5 \mathrm{~s}$ is the onset of the actual movement.

(a)

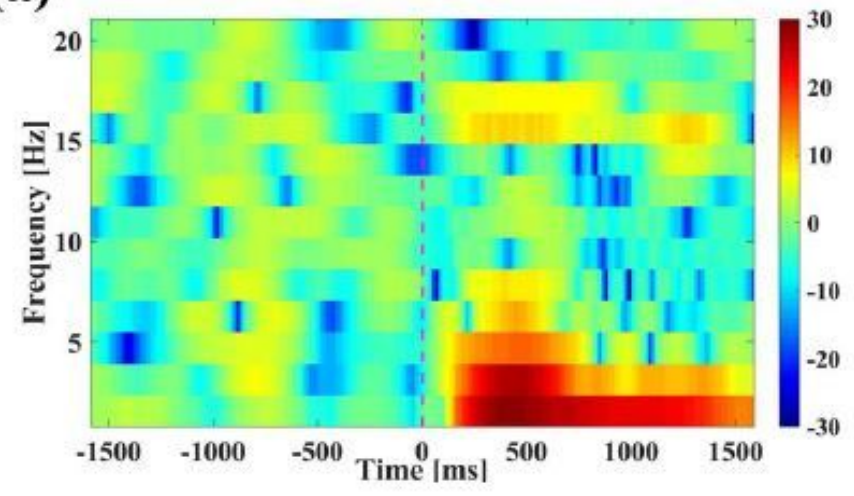

(b)

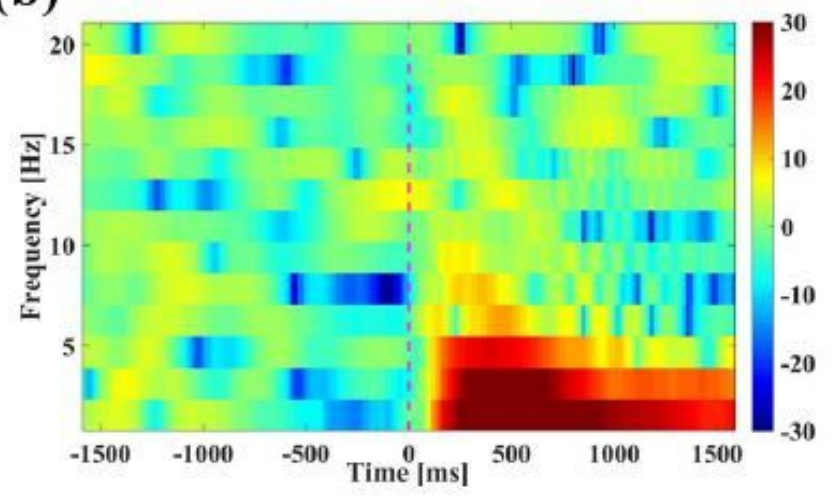

Figure 6 
Time-Frequency plots of movement. (a) Movement under condition WO-OHM and (b) Movement under condition W-OHM. The averaged results are in frequencies [0 16] Hz at Cz channel from $-1.5 \mathrm{~s}$ to $1.5 \mathrm{~s}$ of motion execution. Note that time $0 \mathrm{~s}$ refers to Go-Cue. Note that time $0 \mathrm{~s}$ refers to the time point when the movement cue appears, and time $0.5 \mathrm{~s}$ is the onset of the actual movement.

a)
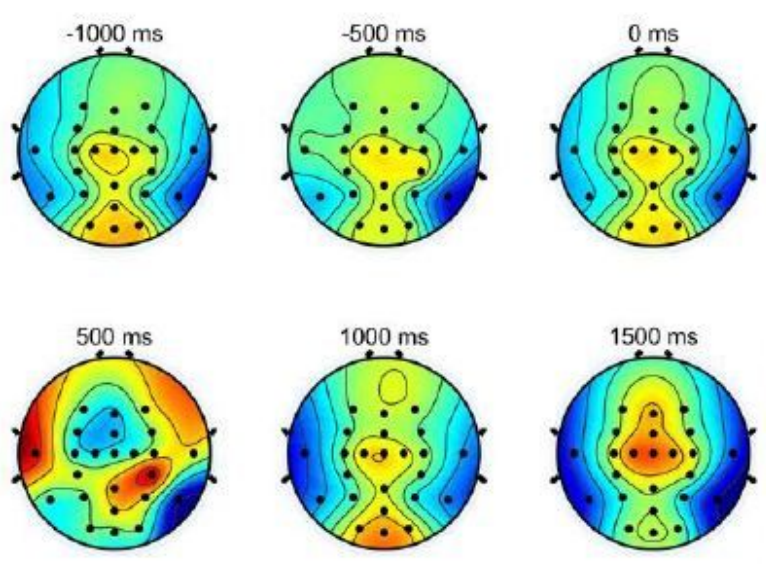

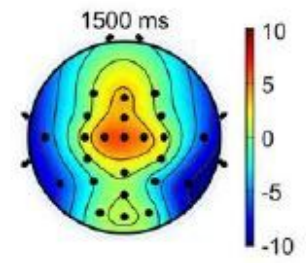

(b)
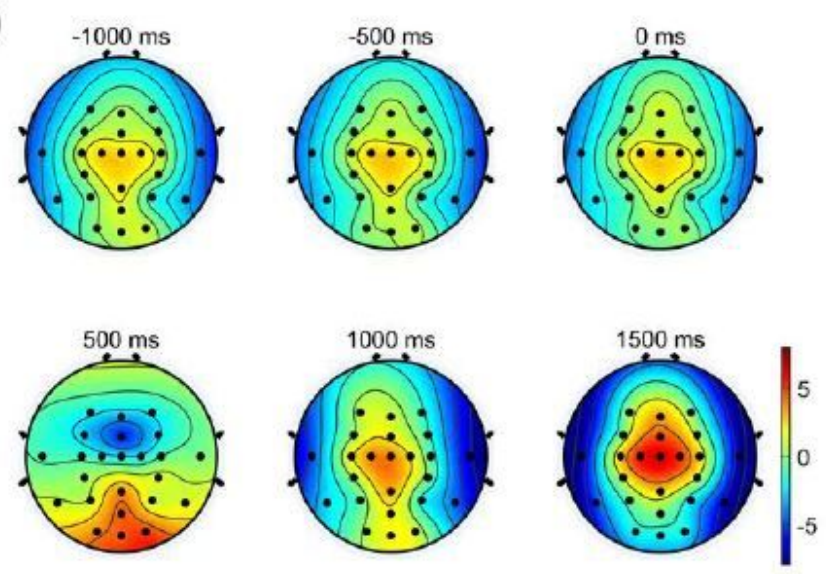

Figure 7

Averaged EEG potential topographic maps of hand movement. (a) Movement under condition WO-OHM and (b) Movement under condition W-OHM. Note that time $0 \mathrm{~s}$ refers to the Go-Cue, and time $0.5 \mathrm{~s}$ is the onset of the actual movement. 


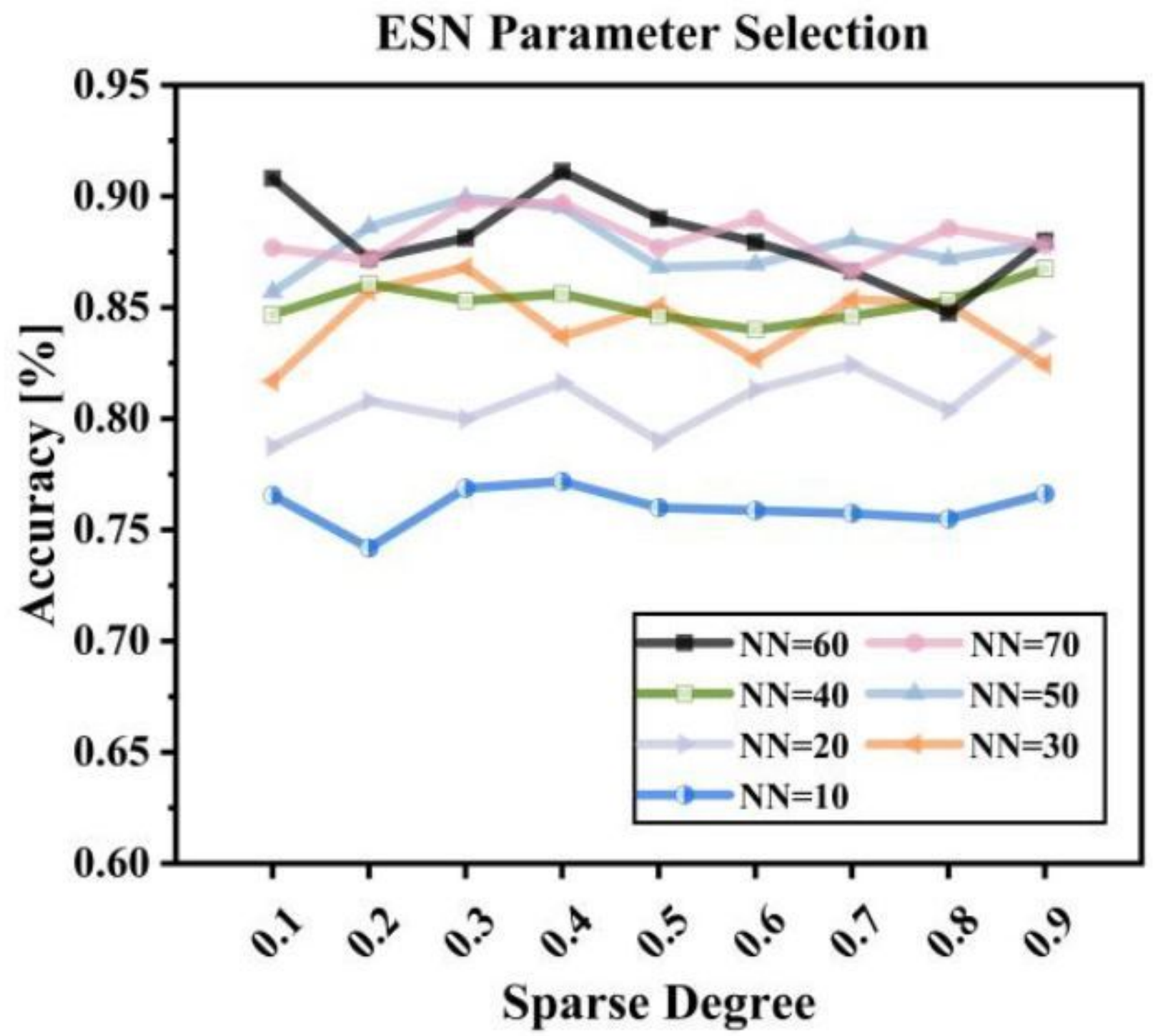

Figure 8

ESN parameter selection with different reservoir sparse degree $\mathrm{c}$ and reservoir size NN. 


\section{Decoding Performance Comparison}

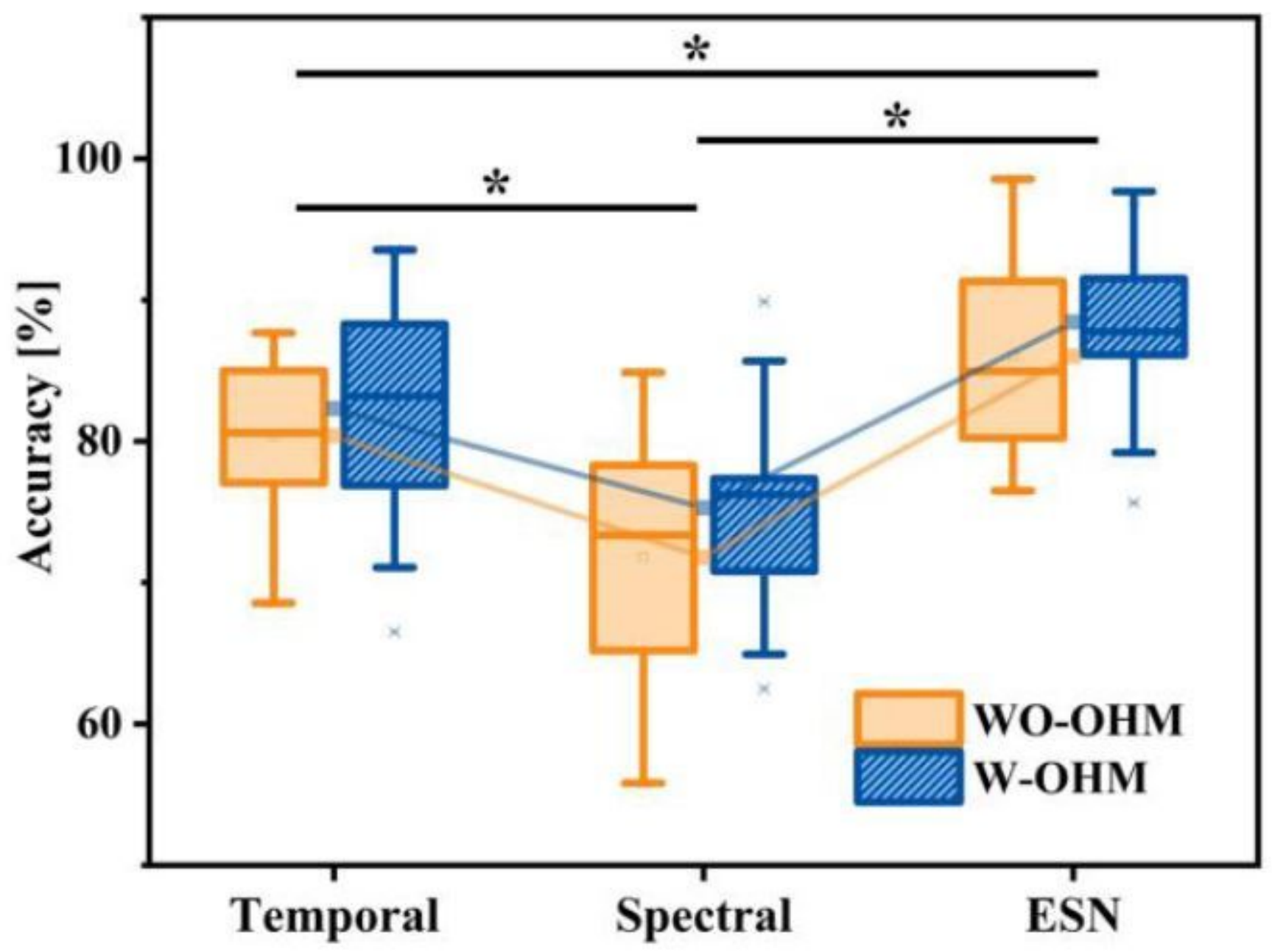

Figure 9

Box-Plots of average decoding accuracy under conditions W-OHM and WO-OHM by using three kinds of features with LDA classifier. The asterisk marks significant differences. The broken lines show the interaction effect of feature type and movement condition on decoding accuracy. 